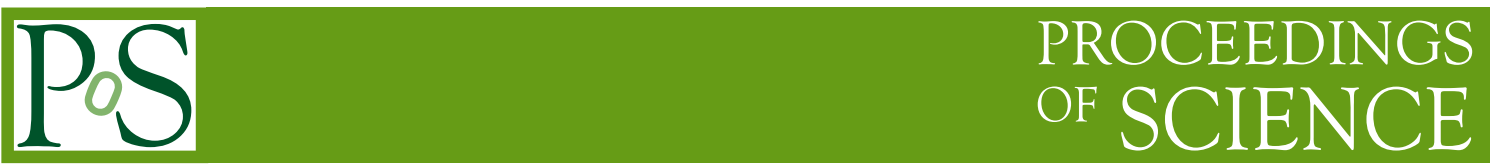

\title{
First results from the AugerPrime engineering array
}

\section{Zizhao Zong $^{* a}$ for the Pierre Auger Collaboration ${ }^{b}$}

anstitut de Physique Nucléaire d'Orsay, IN2P3-CNRS, Université Paris-Sud, Université

Paris-Saclay, 91406 Orsay Cedex, France

${ }^{b}$ Observatorio Pierre Auger, Av. San Martín Norte 304, 5613 Malargüe, Argentina

E-mail: auger_spokespersons@fnal.gov

Full author list: http://www.auger.org/archive/authors_icrc_2017.html

\begin{abstract}
The surface detector array of the Pierre Auger Observatory consists of 1660 water-Cherenkov detectors (WCDs) which sample the charged particles and photons of air showers initiated by cosmic rays of very high energy. With the AugerPrime upgrade, the collaboration aims to increase the particle identification capability of the surface detectors. Scintillator surface detectors (SSDs) will be added above the water-Cherenkov detectors and the stations will be equipped with new electronics having better timing accuracy, higher sampling frequency, and increased processing capability. Furthermore, small photomultipliers will be added to the WCDs to allow for an increase of the dynamic range of the signal readout. In October 2016, an engineering array consisting of 12 AugerPrime detector stations was installed within the existing array of the Observatory.

In this contribution, we will discuss the first results from the AugerPrime engineering array. In particular, the detector calibration in units of vertical equivalent muon (VEM) for WCD and minimum ionizing particle (MIP) for SSD. Furthermore, we will discuss the temperature dependence of the detector parameters, show the lateral distribution function of showers measured with both detector types, and present the preliminary results of the study on signals from doublet stations.
\end{abstract}

35th International Cosmic Ray Conference - ICRC2017

12-20 July, 2017

Bexco, Busan, Korea

* Speaker. 

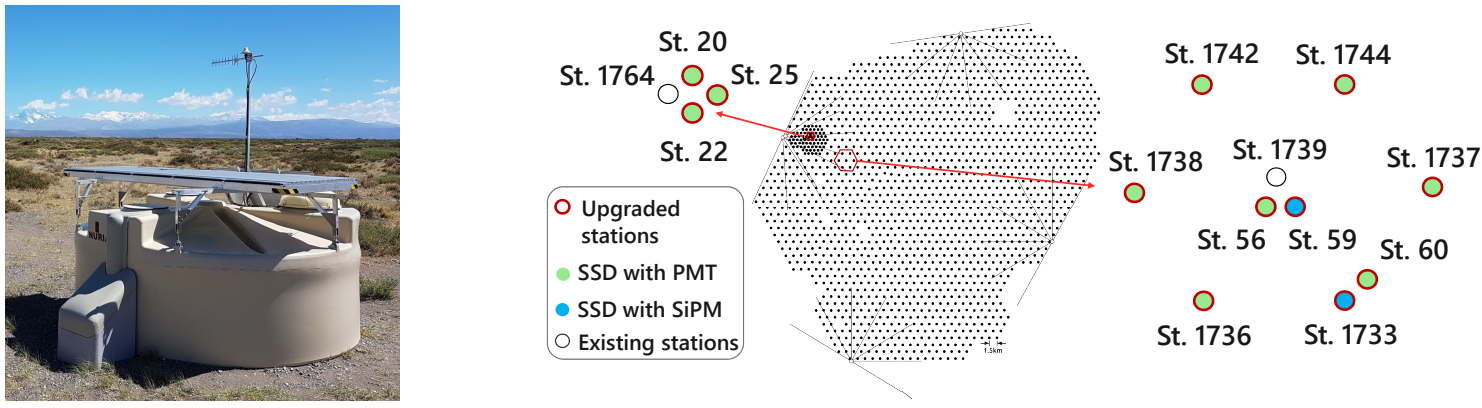

Figure 1: Left: Photograph of an AugerPrime surface detector (St. 56) in operation. Right: Layout of the AugerPrime engineering array. Several groups of multiplet stations are deployed in the EA: stations $(1739,56,59),(1733,60)$, and $(1764,20,22,25)$.

\section{Introduction}

The Pierre Auger Observatory [1], located in western Argentina, near the city of Malargüe in Mendoza province, at a high altitude of $1400 \mathrm{~m}$ a.s.l., aims to probe the origin and characteristics of ultra-high-energy cosmic rays (UHECR). The Observatory combines four fluorescence detector (FD) sites consisting of 27 fluorescence telescopes and a surface detector (SD) array consisting of 1660 water-Cherenkov detectors (WCD), each of which has three 9" photomultiplier tubes (PMT), covering $3000 \mathrm{~km}^{2}$ to achieve a hybrid detection of the Extensive Air Showers (EAS) produced by UHECRs in the atmosphere. In the last decade, several important results have been obtained by the Observatory [2].

By proposing the AugerPrime project [3, 4], the Auger collaboration aims to upgrade the Observatory for shower-by-shower measurements of the mass composition of cosmic rays at the highest energies. The AugerPrime implementation for the SD detectors includes three main elements:

1 Scintillator surface detectors (SSD) of $\sim 3.8 \mathrm{~m}^{2}$ will be mounted above the existing WCDs (see Fig.1 left). Each SSD is composed of 48 extruded plastic scintillator bars, which are read out by wavelength-shifting (WLS) fibers coupled to a single photo-detector [5].

2 Upgraded electronics with better timing accuracy ( $\sim 5 \mathrm{~ns})$, higher sampling frequency (120 $\mathrm{MHz}$ ), and increased processing capability will be employed for upgraded surface detector stations (WCD + SSD) [6].

3 A small PMT (SPMT) will be added to each WCD. It will work together with the three large PMTs to extend the dynamic range of the WCDs [7].

The AugerPrime engineering array (EA) of 12 upgraded detectors was deployed in October 2016 and has been since then continuously taking data. Fig. 1-right shows the layout of AugerPrime EA. Nine upgraded stations are located in a hexagon shape surrounding the existing SD station 1739 in the regular SD array (1500 m spacing). Three upgraded stations are deployed in the so-called AERAlet area near the station 1764 (433 m spacing). Some stations are deployed close to each other (with $11 \mathrm{~m}$ spacing) as doublet or multiplet stations for signal accuracy and other studies (see Fig. 1 right). 

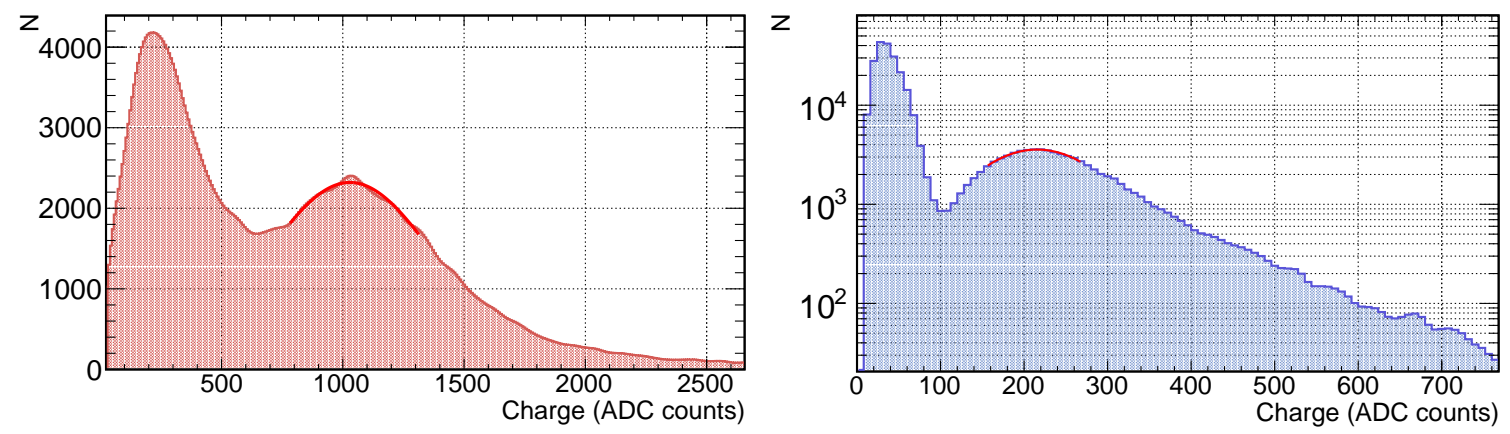

Figure 2: Left: The VEM charge spectrum measured by one of the WCD PMTs. The second peak corresponds to the charge deposited by single muons traversing the detector station. Right: The MIP charge spectrum from the SSD PMT.
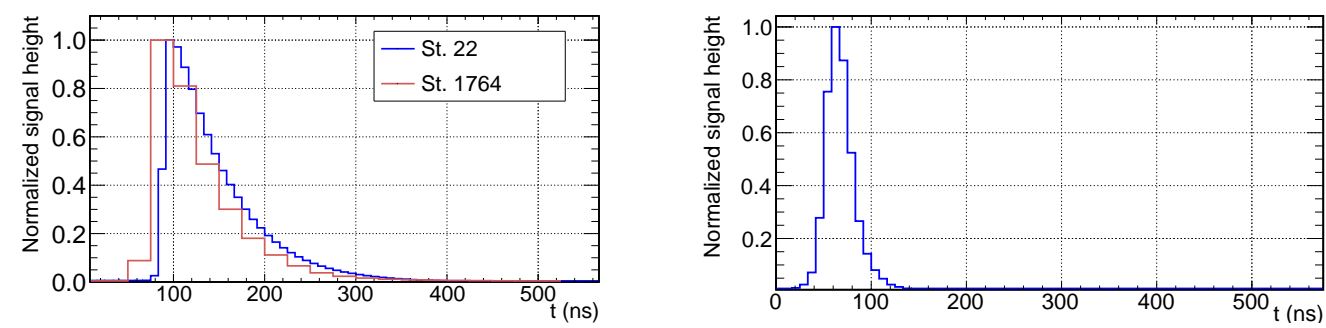

Figure 3: Left: Comparison of muon signal shapes from the existing station 1764 and the upgraded station 22. The signals are normalized by their signal heights. Right: The muon signal shape from a SSD PMT.

In this paper, we present the first results from the AugerPrime engineering array. In particular, we will discuss the calibration and the operation status of WCD and SSD detectors, the shower signals from both detector types, and the signal accuracy obtained with the doublet stations.

\section{Calibration and operation status of the detectors}

In the WCD calibration, the main measured parameter is the average charge deposited by an incident vertical, central muon passing through the WCD [8]. This parameter can be obtained from the charge spectrum of background muons measured by each PMT in the WCD (see Fig. 2-left). In the muon charge spectrum, the second peak is induced by the muons crossing the WCD from all directions. By fitting the peak position, the approximate charge value of a vertical equivalent muon (VEM) can be determined. Based on simulations and measurements with test-detectors, the final VEM charge used in the detector calibration is determined with $Q_{\mathrm{VEM}}=Q_{\mathrm{VEM}}^{\text {raw }} / 1.01$, where 1.01 is the factor of the conversion from omni-directional to vertical muons.

For the SSDs, the charge deposited with a minimum ionizing particle (MIP) is used for the calibration (see Fig. 2-right). The raw MIP charge is obtained by fitting the second peak position in the muon spectrum from SSD and the final MIP charge is determined with $Q_{\text {MIP }}=0.87 Q_{\text {MIP }}^{\text {raw }}$, where 0.87 is determined based on related measurements and simulations [5, 9].

The muon charge spectra from both WCDs and SSDs are stored in the muon buffer of each 
local station and sent to the central data acquisition system (CDAS) every 6 minutes. The signals of shower events from the detectors are calibrated based on the real-time muon spectra.

The average signal shapes of background muons from WCDs and SSDs are also recorded and sent to CDAS (see Fig. 3). Various quantities to evaluate the detector performance can be parameterized from the digitized average signal shapes. The PMTs have a fast response to a single muon, dominated by the Cherenkov light reflected only once at the tank liner. After reaching the peak, the signal exponentially decays due to the losses in consecutive multiple reflections and absorption in water, since the decay time of the muon signals in WCDs is related to the reflection coefficient of the liner and to the transparency of the water in each WCD.

Among the 1660 SD stations, the average value of muon decay time is around $60 \mathrm{~ns}$ (distributed between 50 to $70 \mathrm{~ns}$ ). The area-over-peak (AoP) ratio of the muon signal, proportional to the signal width, is introduced to describe the detector performance and is available from the detector in real-time. Previous studies by the Auger collaboration have shown the long-term evolution of AoP and its importance for detector monitor-

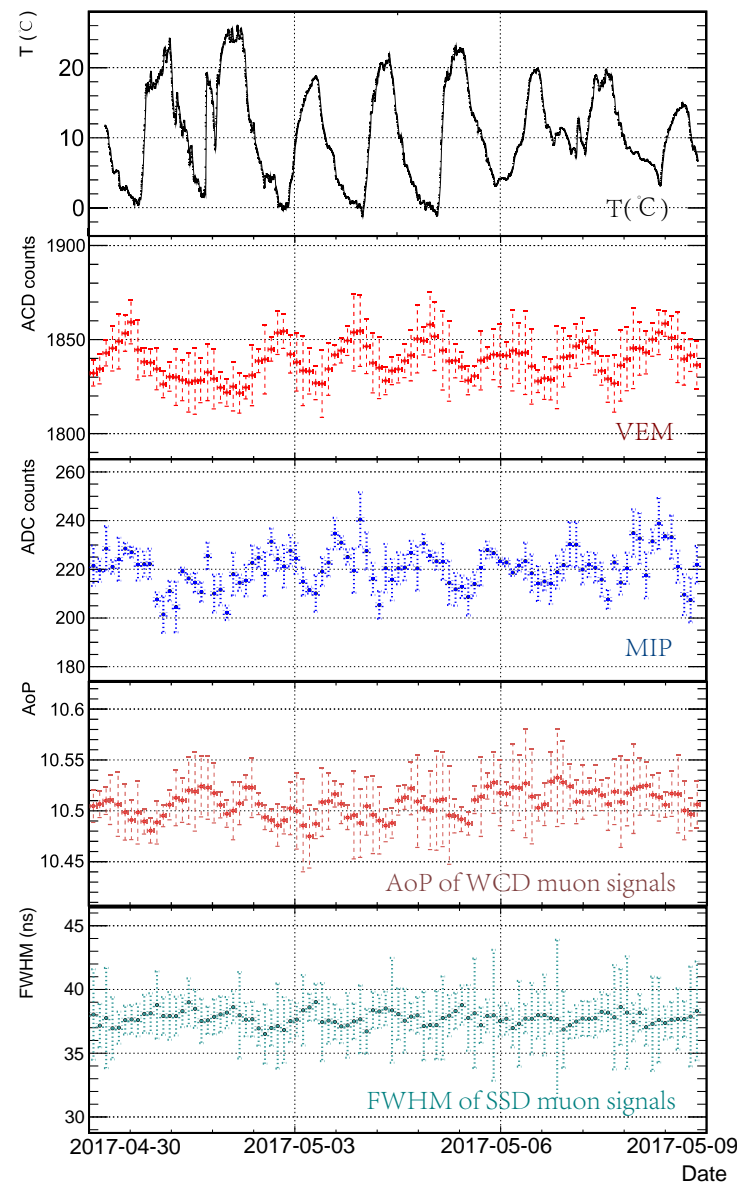

Figure 4: The operation quantities of the detector are affected by the temperature in the field. With the day-to-night temperature fluctuation of $\geq 20^{\circ} \mathrm{C}$, the detector operates in the good stability with an acceptable variation of calibration and signal properties. ing [10]. For the current SD stations, the average value of AoP is around 3.5, or in time units, around $88 \mathrm{~ns}$. In the upgraded stations with new faster electronics $(120 \mathrm{MHz})$ we see essentially the same AoP ratios of around 10.5 which corresponds to $88 \mathrm{~ns}$ in time units.

The muon signal shape in SSD is narrow since the plastic scintillator bars with a thickness of $1 \mathrm{~cm}$ have a fast time response and a good time resolution for the measurement of background muons. In this work, the width (FWHM) of the SSD muon signal is used to monitor the detector performance. From the experimental data, the average value of muon signal width is $\sim 35 \mathrm{~ns}$ for all SSDs.

In the observatory area, the day-night temperature variation is around $20^{\circ} \mathrm{C}$. Fig. 4 shows the related parameters introduced above from the station 20 together with the temperature measured in the CLF (central laser facility) as a function of time over the first week of May 2017. From the plots, we can see that the day-to-night fluctuations of these detector parameters affected by the temperature are: $<3 \%$ for VEM charge, $<5 \%$ for MIP charge, $\sim 1 \%$ for area-over-peak from WCD and $\sim 3 \mathrm{~ns}$ for the FWHM of SSD muon signals. Concerning the WCD, similar variation 

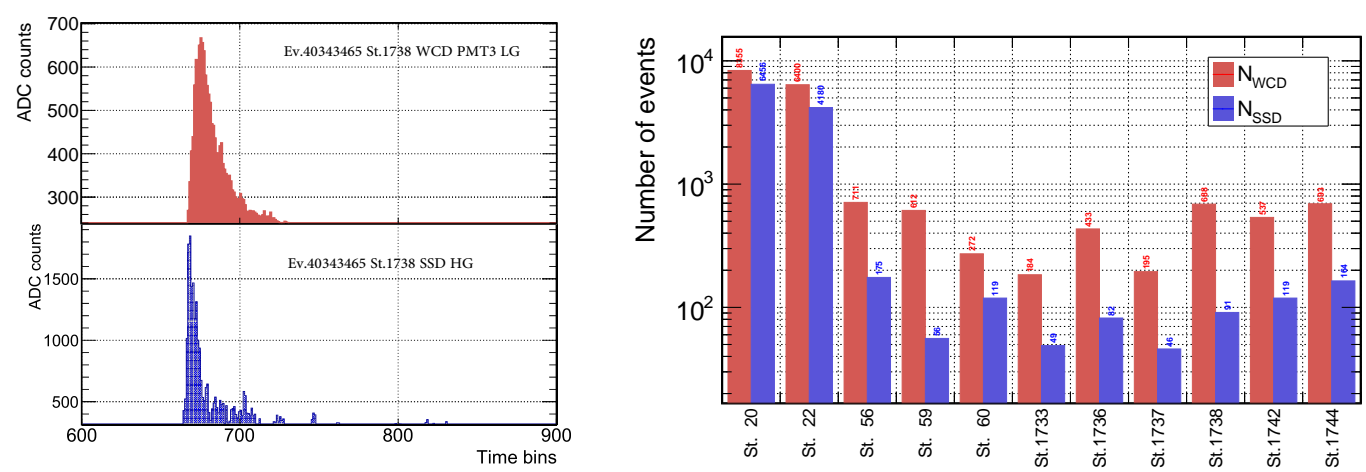

Figure 5: Left: The WCD and SSD signals of station 1738 for the same event. Right: The summary of the event numbers in all EA stations.

was reported for the existing detectors [10]. Since for both detectors, the calibration is performed nearly continuously, the temperature dependence does not affect the data.

\section{Shower signals from AugerPrime stations}

The two detector types have different responses to the electromagnetic (EM) and muonic components of the EAS. Their signals in VEM or MIP units are highly related to the EM energy flux and the muonic flux at ground level. Therefore, signal densities sampled in the same position with WCD and SSD are essential for the determination of the muonic shower component, which is crucial for primary particle identification.

\subsection{Signals from WCD and SSD for detected showers}

Currently, for the detection of shower events in each station the SSD is triggered by the WCD. Fig. 5 shows the signals of WCD and SSD from station 1738 for the same event. The main signal in WCD spreads relatively longer than the one in SSD due to the multiple reflections of the Cherenkov light in the water tank. With the SSD signals, we can clearly see the particles which arrived later than the main flow of secondary particles produced in the EAS development.

During the operation of EA, more than 8000 shower events were recorded. Fig. 5 shows the number of WCD and SSD events in each station. We can see that in the station 20 and 22, which are in the AERAlet area (433 m spacing), have much higher trigger rate than the others, which are deployed in the regular SD array area. As we have been maintaining and optimizing the detectors in the last few months, some of the stations were not working with a full duty cycle. The number of events recorded by EA stations have slight differences but are comparable with the event numbers from stations near by. As the active SSD area is much less than the WCD area (about two fifths), the trigger rate of the shower events for SSDs is relatively less than it is for WCDs. The area of detectors also affects the signal amplitudes of each shower event. For most events, the signal ratio of $S_{\mathrm{SSD}} / S_{\mathrm{WCD}}$ is less than 1 (average ratio around 0.65 ). However, for a given shower, the correlation of $S_{\mathrm{SSD}}$ and $S_{\mathrm{WCD}}$ depends on the distance from the station to the shower axis. The related results are shown in the next section. 
LDF of Ev.163076179300

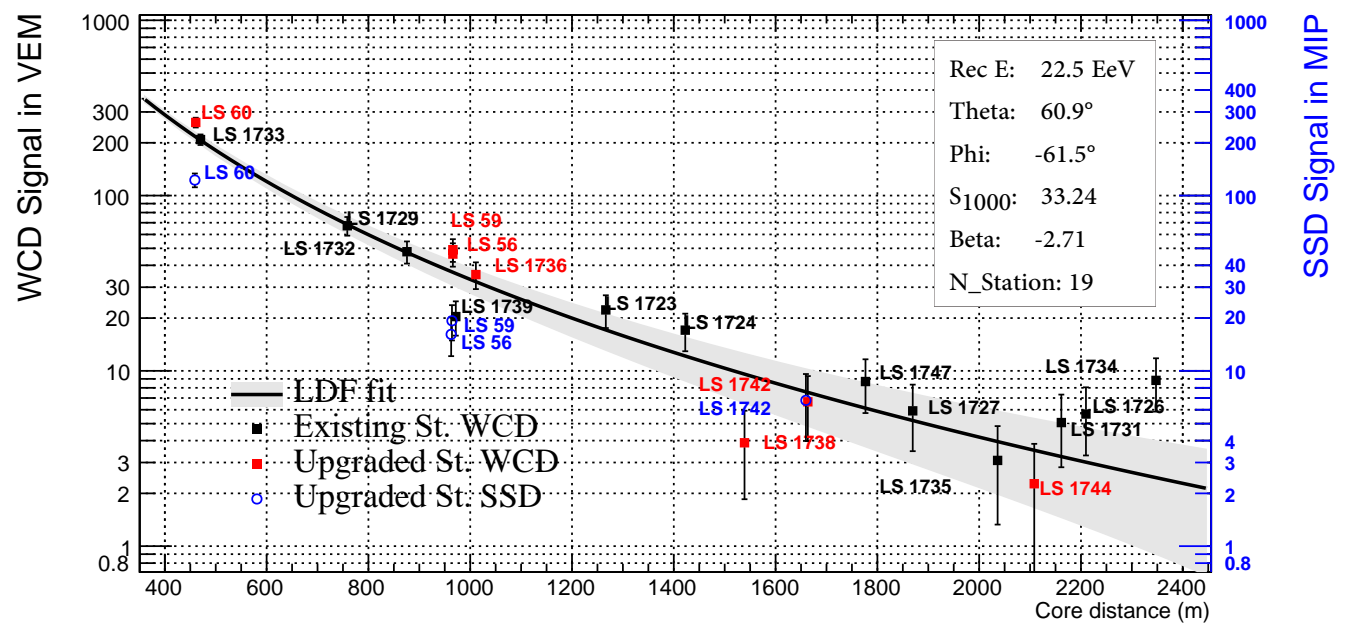

Figure 6: Signals from upgraded local stations (LS) compared to the LDF reconstructed from the existing local stations.

\subsection{Signals from EA stations compared to the lateral distribution function}

The events triggered by the SD array are first selected with the T4 and T5 triggers [11]. The arrival direction is obtained by fitting the start time of each SD signal to a spherical front. The shower core on the ground can be obtained from the fits of the SD signals. The lateral distribution function (LDF) of the air shower can then be described as a modified NKG function

$$
S(r)=S\left(r_{\mathrm{opt}}\right)\left(\frac{r}{r_{\mathrm{opt}}}\right)^{\beta}\left(\frac{r+r_{1}}{r_{\mathrm{opt}}+r_{1}}\right)^{\beta+\gamma}
$$

where $r_{\mathrm{opt}}$ is the reference distance, $r_{1}=700 \mathrm{~m}$ and the $S\left(r_{\mathrm{opt}}\right)$ is an estimation of the shower size. As the spacing of stations is $1500 \mathrm{~m}$ for the SD array, the $r_{\text {opt }}$ is chosen to be $1000 \mathrm{~m}$. For the in-fill array with the $750 \mathrm{~m}$ spacing, the $r_{\text {opt }}$ is $450 \mathrm{~m}$. Note that the parameters $\beta$ and $\gamma$ have some residual dependence on the zenith angle and the shower size.

Fig. 6 shows an example event with a primary energy of $22.5 \mathrm{EeV}$. For this event, 12 existing stations and 7 upgraded stations were triggered. The LDF in the plot is fitted with signals from the existing stations. As can be seen in Fig. 6, WCD signals from upgraded stations are in good agreement with this LDF curve, and SSD signals are relatively lower than WCD signals, as expected.

The global LDF corresponding to the signals from upgraded stations is shown in Fig. 7. Signals from WCDs and SSDs normalized by the shower size are plotted as a function of distance from the station to the shower axis. The LDF of $S_{\mathrm{WCD}}$ from upgraded stations agrees well with the LDF of $S_{\mathrm{WCD} \text {-old }}$ from the existing stations. As expected, the LDF of $S_{\mathrm{SSD}}$ is higher than the LDF of $S_{\mathrm{WCD}}$ in the region close to the shower axis and then gets lower in the region further away. Correspondingly, the ratio of $S_{\mathrm{SSD}} / S_{\mathrm{WCD}}$ is observed to be larger than 1 for the region near the shower axis and then tends to be $\sim 0.4$ at large distances $(>700 \mathrm{~m})$. This can be understood as the effect of the SSD sensitivity to the EM components of the EAS and the relatively smaller area of SSDs. 

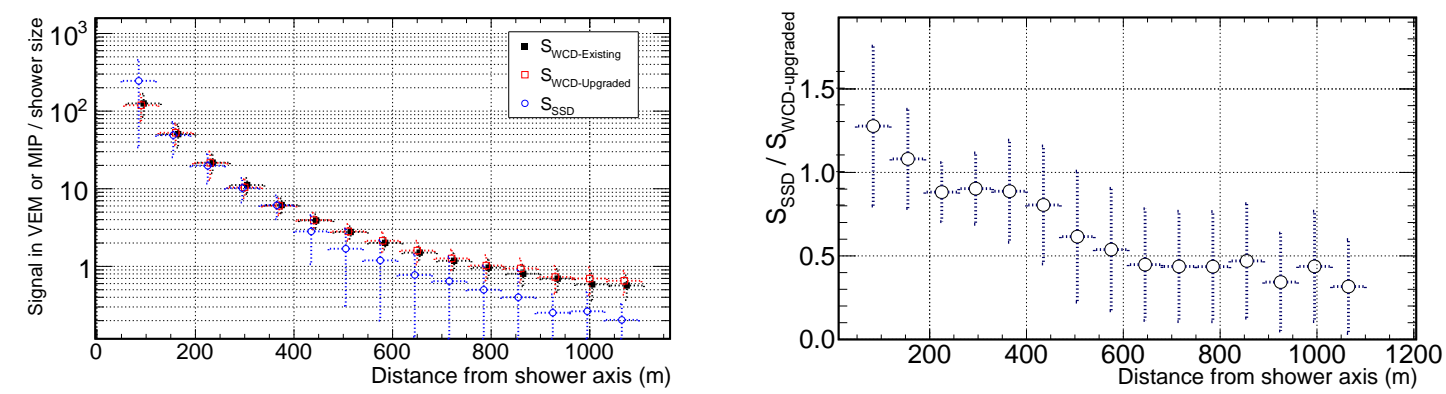

Figure 7: Left: The global LDF of signals from upgraded stations and existing stations. 3084 events reconstructed with the Auger $750 \mathrm{~m}$ array are selected for this analysis (Event selection: T4 events, $\geq 4$ existing stations triggered, no saturation in the stations.) Right: The ratio of $S_{\mathrm{SSD}} / S_{\mathrm{WCD} \text {-upgraded }}$ as a function of distance from the shower axis.
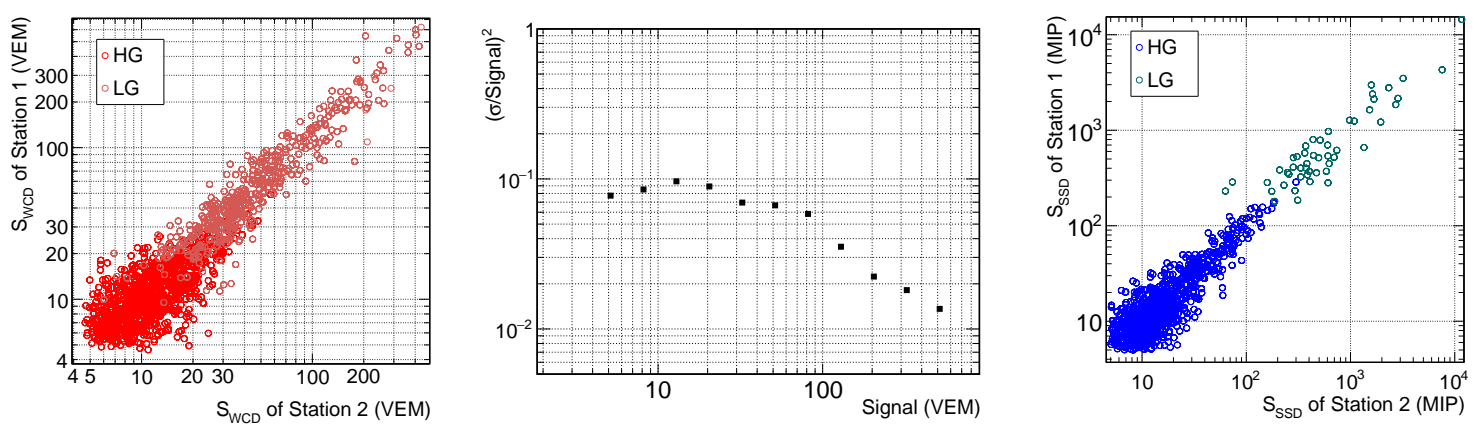

Figure 8: Left and right: The correlations of doublet WCD and SSD signals, respectively. Center: The measured WCD signal accuracy of upgraded stations with respect to the signal amplitude in VEM.

\subsection{Signals from doublet stations}

Some detectors in the EA area are deployed close to each other (spacing $\sim 11 \mathrm{~m}$ ) as doublet or multiplet stations. Since the footprint of a typical EAS extends over several $\mathrm{km}^{2}$, the signals from these multiplet stations can be regarded as measurements in the same point of the shower. The signal correlations of doublets for WCDs and SSDs are shown in Fig. 8. The $S_{\mathrm{WCD}}$ from each station in the doublet is corrected by the LDF to the mean distance of the doublet. This correction can reduce the bias due to the difference of a steep LDF over the $11 \mathrm{~m}$ distance. Good correlation can be seen for $S_{\mathrm{WCD}}$ from both high-gain (HG) and low-gain (LG) channels. For $S_{\mathrm{SSD}}$, therefore, the doublet signals of small amplitudes $(<100 \mathrm{MIP})$ are in good agreement. Currently, there is no LDF fitted to $S_{\mathrm{SSD}}$ available for the doublet signal correction, the signal difference for the large signals (from LG channels) measured near the shower axis is relatively larger than that for the small signals.

A preliminary result from the study of signal accuracy is shown in Fig. 8-center. For the WCD signal of the upgraded stations, the signal accuracy is around $10 \%$, which is comparable to the signal accuracy of the existing stations [12]. Further studies on signal accuracy will be performed with better statistics. 


\section{Summary}

The AugerPrime engineering array has been taking data since October 2016. Detectors are calibrated with the charge of single VEM for $S_{\mathrm{WCD}}$ and single MIP for $S_{\mathrm{SSD}}$. The upgraded stations with WCDs and newly deployed SSDs operate with good stability at the Auger site under a harsh environment with a day-to-night temperature fluctuation of $>20^{\circ} \mathrm{C}$. Signals from EA stations are in a good agreement with the LDF curve fitted with signals from the existing stations. The global LDF for $S_{\mathrm{WCD}}$ from the upgrade stations, normalized by the shower size, agrees well with the results of the LDF fitted to the existing stations. Furthermore, the global LDF of $S_{\mathrm{SSD}}$ shows the SSD sensitivity to the EM components of the EAS as expected. The signals from doublet stations are well correlated and the measured signal accuracy for the WCDs of the upgraded stations is around $10 \%$. Further studies with better statistics are currently under way.

\section{References}

[1] Pierre Auger Collaboration, The Pierre Auger cosmic ray observatory, Nucl. Instrum. Meth. A 798 (2015) 172-213.

[2] M. Unger, for the Pierre Auger Collaboration, Highlights from the Pierre Auger Observatory, these proceedings.

[3] Pierre Auger Collaboration, The Pierre Auger Observatory Upgrade-Preliminary Design Report, (2016) arXiv:1604.03637.

[4] D. Martello, for the Pierre Auger Collaboration, The Pierre Auger Observatory Upgrade, these proceedings.

[5] R. Šmída, for the Pierre Auger Collaboration, Scintillator detectors of AugerPrime, these proceedings.

[6] T. Suomijärvi, for the Pierre Auger Collaboration, Upgraded electronics for AugerPrime, these proceedings.

[7] A. Castellina, for the Pierre Auger Collaboration, The dynamic range of the AugerPrime Surface Detector: technical solution and physics reach, these proceedings.

[8] X. Bertou, for the Pierre Auger Collaboration, Calibration of the surface array of the Pierre Auger Observatory, Nucl. Instrum. Meth. A 568 (2006) 839-846.

[9] D. Schmidt, for the Pierre Auger Collaboration, AugerPrime implementation in the Offline simulation and reconstruction framework, these proceedings.

[10] R. Sato, for the Pierre Auger Collaboration, Long Term Performance of the Surface Detectors of the Pierre Auger Observatory, Proc. Int. Cosmic Ray Conf., Beijing, China, 3 (2011) 204-207.

[11] Pierre Auger Collaboration, Trigger and aperture of the surface detector array of the Pierre Auger Observatory, Nucl. Instrum. Meth. A 613 (2010) 29-39.

[12] P. Bauleo, for the Pierre Auger Collaboration, The accuracy of signal measurement with the water Cherenkov detectors of the Pierre Auger Observatory, Nucl. Instrum. Meth. A 578 (2007) 180-184. 